OPEN ACCESS

Edited by:

Wee-Jun Ong,

Xiamen University, Malaysia

Reviewed by:

Shun Mao,

Tongji University, China

Che Azurahanim Che Abdullah,

Putra Malaysia University, Malaysia

*Correspondence:

Jun-ichi Ozaki

jozaki@gunma-u.ac.jp

Specialty section: This article was submitted to

Carbon-Based Materials,

a section of the journal

Frontiers in Materials

Received: 28 February 2019 Accepted: 16 May 2019

Published: 04 June 2019

Citation:

Ishii T, Horiuchi A and Ozaki J (2019) An Ion-Sensitive Field Effect Transistor

Using Metal-Coordinated

Zeolite-Templated Carbons as a Three-Dimensional Graphene

Nanoribbon Network

Front. Mater. 6:129.

doi: 10.3389/fmats.2019.00129

\section{An lon-Sensitive Field Effect Transistor Using Metal-Coordinated Zeolite-Templated Carbons as a Three-Dimensional Graphene Nanoribbon Network}

\author{
Takafumi Ishii, Akihiro Horiuchi and Jun-ichi Ozaki* \\ Faculty of Science and Technology, International Research and Education Center for Element Science, Gunma University, \\ Kiryu, Japan
}

Zeolite template carbon (ZTC) is a carbon material composed of a three-dimensional network of graphene nanoribbons (GNR), and thus, is expected to exhibit electronic characteristics identical to those of GNR. ZTC is capable of doping heteroatoms in its structure, which offers the advantage of controlling its electronic properties by the presence of the heteroatoms. In this study, we prepared heteroatom-doped ZTCs and applied them to an ion-sensitive field effect transistor (ISFET), for investigating the effect of heteroatom doping on its electronic properties. The ISFET characteristics of un-doped ZTC include bipolarity, whereas oxygen-doped ZTC shows p-type semiconductor characteristics, and nitrogen-doped ZTC shows n-type characteristics. Furthermore, copper and nitrogen co-doped ZTC exhibits high sensor sensitivity (the change of electric conductivity reaches up to 50\%) as a carbon-based ISFET. As a result of the analytical evaluation, a copper-nitrogen complex is formed in the co-doped ZTC, and the electronic interaction between copper and nitrogen atoms is able to change electrochemically. Owing to the presence of the copper-nitrogen complex, the co-doped ZTC shows specific ISFET characteristics.

Keywords: zeolite-templated carbon, doped carbon, graphene nanoribbon, ion sensor, ISFET

\section{INTRODUCTION}

An ion-sensitive field effect transistor (ISFET) is expected to be a novel sensor device for electrically detecting ions and has been applied to biosensors (Mao et al., 2017; Chen et al., 2018, 2019; Syu et al., 2018), pH sensors (Duroux et al., 1991), etc. Silicon based materials are generally used as the channel material of the ISFET (Martinoia et al., 2005); however, it has an issue in that the sensitivity is lowered by an oxide film formed on its surface. Graphene nanoribbon (GNR) has attracted attention as a channel material to replace silicon (Ahmadi et al., 2016). GNR is a graphene sheet with its width reduced to the order of nanometers. Unlike graphene, GNR shows high sensitivity as a channel material of ISFET because of having a band gap (Zhang and Cui, 2013). The electronic state of GNR is influenced by the edge sites of the graphene sheet and the doping of heteroatoms. This is explained by the localization of $\pi$ electrons to the edge sites (Bronner et al., 2012) and the electron attraction/donation action (Yu et al., 2010; Wang et al., 2014) in the $\pi$ electron system 
caused by the presence of heteroatoms. These phenomena make it possible to control the electronic properties of GNR such as by managing its band gap formation or changing its Dirac point (Wang et al., 2014). Although GNR is considered to be a promising channel material to overcome the shortcomings of silicon, there are many problems associated with its practical application as an ISFET. For synthesis of GNR, precise and high-cost techniques are required, such as an electron beam lithography (Bai and Huang, 2010) and carbon nanotube incision (Kosynkin et al., 2009). In addition, GNR has a problem in that the current flowing in the channels is too small, even as compared with graphene and silicon, because of its smaller channel area.

We focused on zeolite-templated carbon (ZTC) (Nishihara and Kyotani, 2018) as a novel carbon material for replacing GNR in this study. ZTC is composed of a three-dimensional network of GNR with a width of approximately $1 \mathrm{~nm}$ and the pore size is uniform at approximately $1.2 \mathrm{~nm}$ (Nishihara and Kyotani, 2018). Because of its three-dimensional network of GNR, ZTC is expected to increase the detected current value in ISFET, while maintaining the same sensitivity as GNR. ZTC does not require expensive processes such as lithography for its synthesis and can be industrially manufactured at a low cost. Moreover, GNR is obtained by a "top-down" approach of chopping graphene, whereas ZTC is prepared by a "bottom-up" approach of template carbonization using a chemical vapor deposition (CVD) method. When doping heteroatoms in GNR, structural geometries are easily changed. However, in the case of ZTC, it is possible to freely select a carbon source for template carbonization, and by using the source containing heteroatoms, doping of heteroatoms can be achieved while the structure is controlled. This means that ZTC has the possibility of developing various electronic properties due to heteroatom-doping. Till date, ZTC has reportedly been applied to electrochemical electrodes (Itoi et al., 2011, 2014, 2016; Berenguer-Murcia et al., 2013; Nueangnoraj et al., 2015) and gas storage materials (Nishihara et al., 2014; Itoi et al., 2017); however, its electronic states or semiconductor characteristics have not been reported. Similar to GNR, if ZTC functions as an ISFET, ZTC can be used as a novel industrially applicable carbon material having GNR characteristics.

In this study, we investigated the possibility of ZTC composed of a GNR three-dimensional network as a novel channel material of graphene for ISFET channel material. ZTC doped with typical and transition metal elements was prepared, and the influence of the presence of these heteroatoms on ISFET characteristics was evaluated. We tried to clarify the principle of the heteroatoms involved in ISFET characteristics of ZTC by investigating the chemical state of them.

\section{EXPERIMENTAL}

\section{Sample Preparation}

\section{Preparation of ZTC and Oxidized ZTC}

ZTC was synthesized using Y-type zeolite (HSZ-320NAA, Na cation type, Tosoh Corp.) as a template. The zeolite was vacuumdried at $150^{\circ} \mathrm{C}$ for $48 \mathrm{~h}$. Furfuryl alcohol (FA) was added dropwise to the dried zeolite powder, and then left standing overnight at room temperature under vacuum. After the excess FA was washed with mesitylene approximately 5 times, a FA/zeolite composite was obtained by suction filtration. This composite was heat-treated at $80^{\circ} \mathrm{C}$ for $48 \mathrm{~h}$ under nitrogen flow, and then heat-treated at $150^{\circ} \mathrm{C}$ for $8 \mathrm{~h}$. Thereafter, the composite was carbonized by heating it to $800^{\circ} \mathrm{C}$ under nitrogen flow. Propylene ( 2 vol.\% in $\mathrm{N}_{2}$ ) was allowed to flow for $4 \mathrm{~h}$ while maintaining the temperature at $800^{\circ} \mathrm{C}$, and a further carbon source was filled in the zeolite pores to obtain a carbon/zeolite composite. The obtained composite was subjected to wet pulverization treatment in agate mortar with methanol for $30 \mathrm{~min}$. The zeolite template included in the composite was removed with 47 wt. \% hydrofluoric acid to obtain a ZTC sample. The obtained ZTC with FA and propylene as a carbon source is named P-ZTC.

For preparing oxygen doped ZTC, P-ZTC was sandwiched between gold mesh electrodes, and an electrochemical oxidation treatment was carried out using a three-electrode cell. A reversible hydrogen electrode (RHE) was used for the reference electrode, Pt was used for the counter electrode, a sample electrode was used for the working electrode, and $0.5 \mathrm{~mol}$ $\mathrm{L}^{-1} \mathrm{H}_{2} \mathrm{SO}_{4}$ was used for the electrolyte. The electrochemical oxidation treatment was carried out by maintaining the working electrode potential at $2.5 \mathrm{~V}$ vs. the RHE for $200 \mathrm{~s}$. The sample after the electrochemical oxidation is called Ox-ZTC.

\section{Preparation of Nitrogen-Doped ZTC}

Acetonitrile $(\mathrm{ACN})$ was added dropwise to the dried zeolite powder, and left standing overnight at room temperature under vacuum. After washing the excess ACN with mesitylene approximately 5 times, an ACN/zeolite composite was obtained by suction filtration. Heat treatment was performed at $60^{\circ} \mathrm{C}$ for $48 \mathrm{~h}$ under a nitrogen flow, and then the $\mathrm{ACN}$ was carbonized by heating it to $800^{\circ} \mathrm{C}$ under a nitrogen flow. While maintaining the temperature of $800^{\circ} \mathrm{C}, \mathrm{ACN}\left(3 \mathrm{vol} . \%\right.$ in $\mathrm{N}_{2}$ ) was passed for $6 \mathrm{~h}$. The zeolite template was removed by the same operation as P-ZTC to obtain a nitrogen-doped ZTC sample, called N-ZTC.

\section{Preparation of Copper-Doped ZTC}

Sodium ions are present as exchange cations for the zeolite used as a template. This exchange cation was ion-exchanged to $\mathrm{Cu}^{2+}$ as follows. Ion exchange was carried out by dispersing $5 \mathrm{~g}$ of the zeolite in $100 \mathrm{ml}$ of $0.3 \mathrm{~mol} \mathrm{~L}^{-1}$ aqueous solution of $\mathrm{CuCl}_{2}$, and stirring at $90^{\circ} \mathrm{C}$ for $1 \mathrm{~h}$ using a hot stirrer. The ionexchanged zeolite was suction-filtered and washed with distilled water. The pristine zeolite, a white powder, turns blue by copper ion exchange. Using the prepared ion-exchanged zeolite as a template, a ZTC sample was prepared in the same manner as mentioned in section Preparation of ZTC and oxidized ZTC, and is named Cu-ZTC. Nitrogen-doped ZTC samples were also prepared using the ion-exchanged zeolite as a template according to the method in section Preparation of nitrogen-doped ZTC, and are denoted as $\mathrm{Cu}-\mathrm{N}-\mathrm{ZTC}$.

\section{Characterization}

\section{Structural Analysis}

The crystal structures of the prepared ZTC samples were evaluated using X-ray diffraction (XRD, XRD-6100, Cu-Ka, SHIMADZU Corp.). In order to evaluate the specific surface 
area and pore structure of the sample, nitrogen adsorption measurement at $-196^{\circ} \mathrm{C}$ was carried out using an automatic surface area analyzer (BELSORP MAX, Microtrac BEL Corp.). As a pretreatment, the sample was heat-treated at $150^{\circ} \mathrm{C}$ for $2 \mathrm{~h}$ under vacuum. From the resulting nitrogen adsorption isotherm, the specific surface area was determined by applying the Brunauer-Emmett-Teller (BET) theory. The range of the BET plot was $\mathrm{P} / \mathrm{P}_{0}=0.01$ to 0.05 . The total pore volume of each sample was obtained from the nitrogen adsorption amount of $\mathrm{P} / \mathrm{P}_{0}=0.98$. The pore size distribution curves and micropore volumes were obtained by the density functional theory (DFT) and MP methods, respectively. The microstructure and element distribution of the sample were evaluated by high-angle annular dark-field scanning transmission microscopy (STEM-HAADF) and energy dispersive X-ray spectroscopy (EDS) measurement. A single atom analysis electron microscope (JEM-ARM $200 \mathrm{~F}$, JEOL Corp.) was used for the measurement.

\section{Temperature Programmed Desorption}

Temperature-programmed desorption (TPD) measurement was carried out to investigate the amount of edge sites and the element composition of the ZTC samples. Approximately $1 \mathrm{mg}$ of the sample was placed in a graphite sample holder and transferred into a TPD apparatus. The inside of the apparatus was evacuated to a $10^{-5} \mathrm{~Pa}$ level. The sample was heated up to $1,600^{\circ} \mathrm{C}$ at a heating rate of $10^{\circ} \mathrm{C} \mathrm{min} \mathrm{m}^{-1}$ by high frequency induction heating, and the desorbed gases were quantitatively analyzed with a quadrupole mass spectrometer (MPH-100 M, Inficon Co., Ltd.). From the components and amounts of desorbed gases, the amount of edge sites and element composition were determined. The details of TPD measurement have been reported in previous studies (Ishii et al., 2014, 2017a).

\section{X-Ray Photoelectron Spectroscopy}

In order to obtain information on the surface chemical state of the sample, X-ray photoelectron spectroscopy (XPS) measurement was carried out using Kratos AXIS NOVA (SHIMADZU Corp.). Al K $\alpha$ radiation (10 mA, $15 \mathrm{kV}$ ) was used as an X-ray source. The charge-up correction was performed by setting the peak position of the $\mathrm{C} 1 \mathrm{~s}$ spectrum to $284.5 \mathrm{eV}$.

\section{Atomic Absorption Spectrometry}

The amount of metal species present in the sample was quantified by atomic absorption analysis (AAS). The sample was incinerated by oxygen plasma treatment and dissolved in $0.5 \mathrm{~mol} \mathrm{~L} \mathrm{~L}^{-1}$ $\mathrm{HNO}_{3}$ aqueous solution to prepare a measurement solution. The concentration of metal ions in the solution was measured using an atomic absorption spectrophotometer (iCE3300, Thermo Fisher Scientific, Inc.). The amount of metal in the sample was calculated from the determined metal ion concentration.

\section{Work Function Measurement}

In order to compare the Fermi level of the sample, a contact potential difference (CPD) was measured using a Kelvin probe (UVH-KP020-50, KP Technology Co., Ltd.), and a work function was also calculated. The sample was filled in a sample base made of aluminum, and measurement was carried out at ambient temperature and atmospheric pressure in the air. Au powder (particle diameter 1-2 $\mu \mathrm{m}, 99.99 \%$, Nilaco Corp.) was used as a reference sample. The work function of $\mathrm{Au}$ powder was set to $5.1 \mathrm{eV}$ (Kahn, 2016). The work function of the sample was obtained from the difference between the obtained CPDs for Au and the sample.

\section{Evaluation of Ion Sensing Characteristics}

Glassy carbon plates were used for the source and drain electrodes for the ISFET. The ZTC sample was sandwiched between two glassy carbon electrodes and fixed with an acrylic plate and a plastic screw to fabricate a ZTC-ISFET. Carbon black (VXC, VXC72R, Cabot Co., Ltd.) was also evaluated in the same manner, as a reference sample. Activated carbon (YP-50F, Kuraray Chemical Co., Ltd.) was used for the gate electrode. The transistor characteristics of the ISFET in $1 \mathrm{~mol} \mathrm{~L}^{-1} \mathrm{HCl}$ aqueous solution were evaluated by a semiconductor characterization system (4200-SCS, KEITHLEY Corp.). The source-drain voltage $\mathrm{V}_{\mathrm{DS}}$ was fixed to $0.01 \mathrm{~V}$, and the source-gate voltage $\mathrm{V}_{\mathrm{GS}}$ was applied from -0.5 to $0.5 \mathrm{~V}$ with $0.1 \mathrm{~V}$ steps. In each $\mathrm{V}_{\mathrm{GS}}$, the current flowing between the source and drain electrodes when reaching the ion adsorption equilibrium, $\mathrm{I}_{\mathrm{DS}}$, was recorded. When $\mathrm{V}_{\mathrm{GS}}$ is negative, the change of $\mathrm{I}_{\mathrm{DS}}$ by $\mathrm{V}_{\mathrm{GS}}$ is measured with reference to the value of $\mathrm{I}_{\mathrm{DS}}$ obtained at $\mathrm{V}_{\mathrm{GS}}=-0.1 \mathrm{~V}$, and the value of $\mathrm{I}_{\mathrm{DS}}$ obtained at $\mathrm{V}_{\mathrm{GS}}=0.1 \mathrm{~V}$ is also used as a reference in a positive $\mathrm{V}_{\mathrm{GS}}$ region (as shown in Figure $\mathrm{S} 1$ ).

\section{RESULTS AND DISCUSSION}

\section{Structural Evaluation of ZTC Samples}

Table 1 shows elemental compositions obtained from TPD and AAS measurements of each ZTC sample. The hydrogen contents confirmed in the samples are higher than that of the various carbon materials such as a carbon black (Kashihara et al., 2012; Ishii et al., 2014; Hoshikawa et al., 2016), indicating that the ZTC samples have a large number of edge sites. The hydrogen content of P-ZTC is $16.2 \%$, which is the maximum value in all samples, but the oxygen content is as small as $1 \%$, indicating that most of the edge sites terminated with hydrogen. The oxygen content of Ox-ZTC subjected to electrochemical oxidation is $3.6 \%$, more than three times that of P-ZTC. As the amount of hydrogen in Ox-ZTC is reduced to approximately $60 \%$ of that of

TABLE 1 | Elemental compositions of zeolite-templated carbon (ZTC) samples determined from temperature-programmed desorption (TPD) and atomic absorption analysis (AAS).

\begin{tabular}{lccccc}
\hline Sample & \multicolumn{5}{c}{ Atomic concentration/\% } \\
\cline { 2 - 6 } & $\mathbf{C}$ & $\mathbf{N}$ & $\mathbf{0}$ & $\mathbf{H}$ & ${ }^{*} \mathbf{1} \mathbf{C u}$ \\
\hline P-ZTC & 82.8 & 0 & 1.0 & 16.2 & 0 \\
Ox-ZTC & 86.3 & 0 & 3.6 & 10.1 & 0 \\
N-ZTC & 82.8 & 2.4 & 3.1 & 11.7 & 0 \\
Cu-ZTC & 84.9 & 0 & 3.0 & 12.1 & 0.27 \\
Cu-N-ZTC & 82.4 & 3.0 & 2.5 & 12.1 & 0.04 \\
\hline
\end{tabular}

* 1 Cu concentrations were determined by atomic absorption spectrometry. 


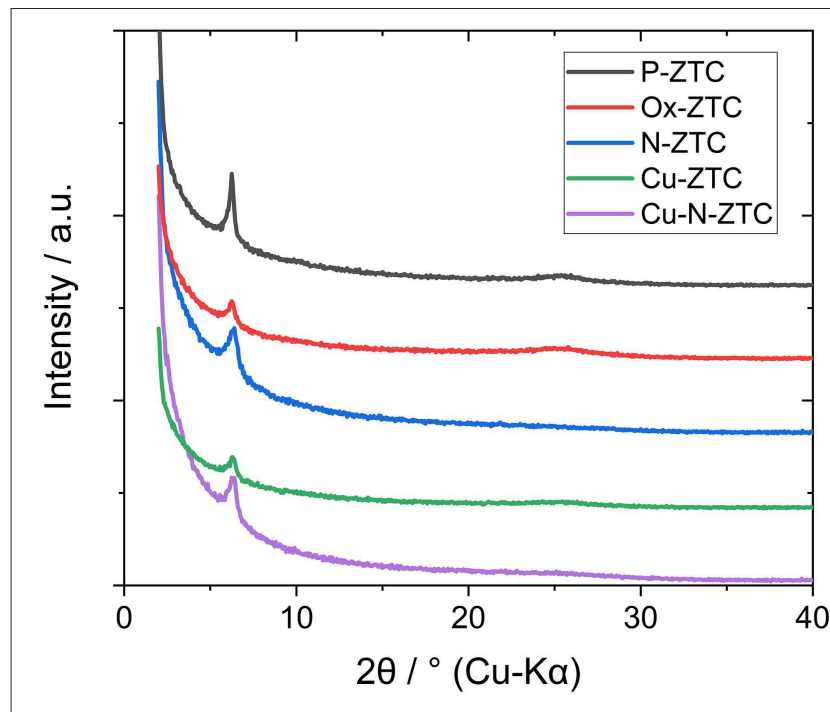

FIGURE 1 | XRD patterns of ZTC samples.

P-ZTC, it is expected that the edge hydrogen is oxidized by the electrochemical oxidation of P-ZTC, and that oxygen-containing functional groups are formed. The nitrogen content of N-ZTC $(2.4 \%)$ is a common value regarded as a doping amount for nitrogen-doped carbons (Ozaki et al., 2006; Kan-Nari et al., 2010; Deng et al., 2016; Ishii et al., 2017b). In the sample doped with $\mathrm{Cu}$, the presence of $\mathrm{Cu}$ was confirmed, although it was a trace amount. The zeolite was removed with hydrofluoric acid while the sample preparation, $\mathrm{Cu}$ as confirmed here, is considered to be incorporated in the carbon structure in an insoluble state by the acid.

Figure 1 shows the X-ray diffraction patterns of the ZTC sample obtained from the XRD measurement. A single peak was observed at $2 \theta=6.3^{\circ}$ for all samples, derived from the (111) plane of the zeolite template, showing high structural regularity (Nishihara and Kyotani, 2018). Although the peak of the heteroatom-doped sample is weaker than that of the undoped sample (P-ZTC), the peak is clearly confirmed in them, indicating that the degradation of structural regularity owing to the doping level is relatively small. The nitrogen adsorption isotherm of the sample and the pore size distribution obtained by the DFT method are shown in Figure S2. The obtained isotherms are classified as Type I and indicate that the samples are micro porous carbon with a sharp pore size distribution of approximately $1.2 \mathrm{~nm}$. Table 2 shows the BET surface area, total pore, and micro pore volumes obtained from the nitrogen adsorption isotherm. The heteroatom-doped samples have a reduced specific surface area as compared to P-ZTC. This decrease in specific surface area is caused by the degradation of the uniform pore structure estimated from the result of XRD. Regarding $\mathrm{Cu}-\mathrm{N}-\mathrm{ZTC}$, the existing form of $\mathrm{Cu}$ was evaluated by STEM-HAADF observation. Element mapping of $\mathrm{C}, \mathrm{N}, \mathrm{O}$, and $\mathrm{Cu}$ of $\mathrm{Cu}-\mathrm{N}-\mathrm{ZTC}$ was performed, as shown in Figure 2. From the STEM-HAADF images, it was observed that not only were $\mathrm{O}$ and $\mathrm{N}$ uniformly dispersed atomically in the sample, but also $\mathrm{Cu}$.
TABLE 2 | Specific surface area and pore volumes of ZTC samples.

\begin{tabular}{|c|c|c|c|}
\hline Sample & $\mathrm{S}_{\mathrm{BET}} / \mathrm{m}^{2} \mathrm{~g}^{-1}$ & $\mathrm{~V}_{\text {total }} / \mathrm{cm}^{3} \mathrm{~g}^{-1}$ & $\mathrm{~V}_{\text {micro }} / \mathrm{cm}^{3} \mathrm{~g}^{-1}$ \\
\hline P-ZTC & 4,080 & 1.93 & 1.82 \\
\hline Ox-ZTC & 2,860 & 1.35 & 1.23 \\
\hline N-ZTC & 3,620 & 1.79 & 1.64 \\
\hline Cu-ZTC & 2,130 & 1.51 & 0.97 \\
\hline CU-N-ZTC & 3,020 & 1.83 & 1.57 \\
\hline
\end{tabular}

\section{Ion Sensing Characteristics of P- Ox- and $\mathrm{N}$-ZTCs}

Figure $3 \mathrm{~A}$ shows the results of the $\mathrm{V}_{\mathrm{GS}}-\mathrm{I}_{\mathrm{DS}}$ plot of P-ZTC and VXC. When VXC was used as a channel material, IDS showed a constant value without respect to the gate voltage. VXC is considered to be a conductor, because no change in conductivity is observed regardless of the presence or absence of ion adsorption. In contrast, P-ZTC shows a semiconductor characteristic. Its $I_{D S}$ varies with respect to $V_{G S}$, indicating that P-ZTC shows ISFET characteristics. The ISFET characteristics show bipolarity, and this result is similar to that of GNR (Zhang and Cui, 2013). This means that P-ZTC has the same semiconductor characteristics as GNR. Therefore, ZTC is considered to be composed of aggregates of GNR, and this is also supported by the fact that a large number of edge sites exist in the structure, as shown in Table 1. Localization of electrons on this large number of edge sites makes the band gap similar to GNR, and as a result, P-ZTC would show such semiconductor characteristics.

Figure $3 \mathrm{~B}$ shows the results of the $\mathrm{V}_{\mathrm{GS}}-\mathrm{I}_{\mathrm{DS}}$ plot of the heteroatom-doped ZTC samples. P-ZTC showed a slight increase in electrical conductivity in both positive and negative directions, indicating bipolarity, but in Ox-ZTC doped with oxygen, the electric conductivity increases with decreasing $\mathrm{V}_{\mathrm{GS}}$. The $\mathrm{I}_{\mathrm{DS}}$ change (sensing sensitivity) of Ox-ZTC is approximately $8 \%$ $\left(\Delta \mathrm{I}_{\mathrm{DS}} / \Delta \mathrm{V}_{\mathrm{GS}}=16 \% / \mathrm{V}\right)$ at the maximum, which is close to the reported value when using GNR as a channel material in ISFET (Zhang and Cui, 2013). By contrast, the electrical conductivity of $\mathrm{N}$-ZTC increases with increasing $\mathrm{V}_{\mathrm{GS}}$. These results show that doping oxygen to ZTC has p-type semiconductor characteristics and doping nitrogen to ZTC has n-type semiconductor characteristics, indicating that controlling a valence state of ZTC is possible by selection of a doping species.

For a deeper understanding, it is important to consider the factors that changed the ISFET characteristics by the doping of heteroatoms. Table 3 shows the work function of each sample. The work function is the energy difference between the Fermi level and the vacuum level. The Fermi level becomes low energy as the work function increases. The work function increased and decreased, respectively in Ox-ZTC N-ZTC as compared to un-doped P-ZTC. This means that by doping with oxygen or nitrogen, the Fermi level of ZTC decreased and increased, respectively. The ISFET characteristics of Ox-ZTC and N-ZTC can be explained by the Fermi level. The Fermi level of OxZTC is close to the valence band, while that of N-ZTC is 

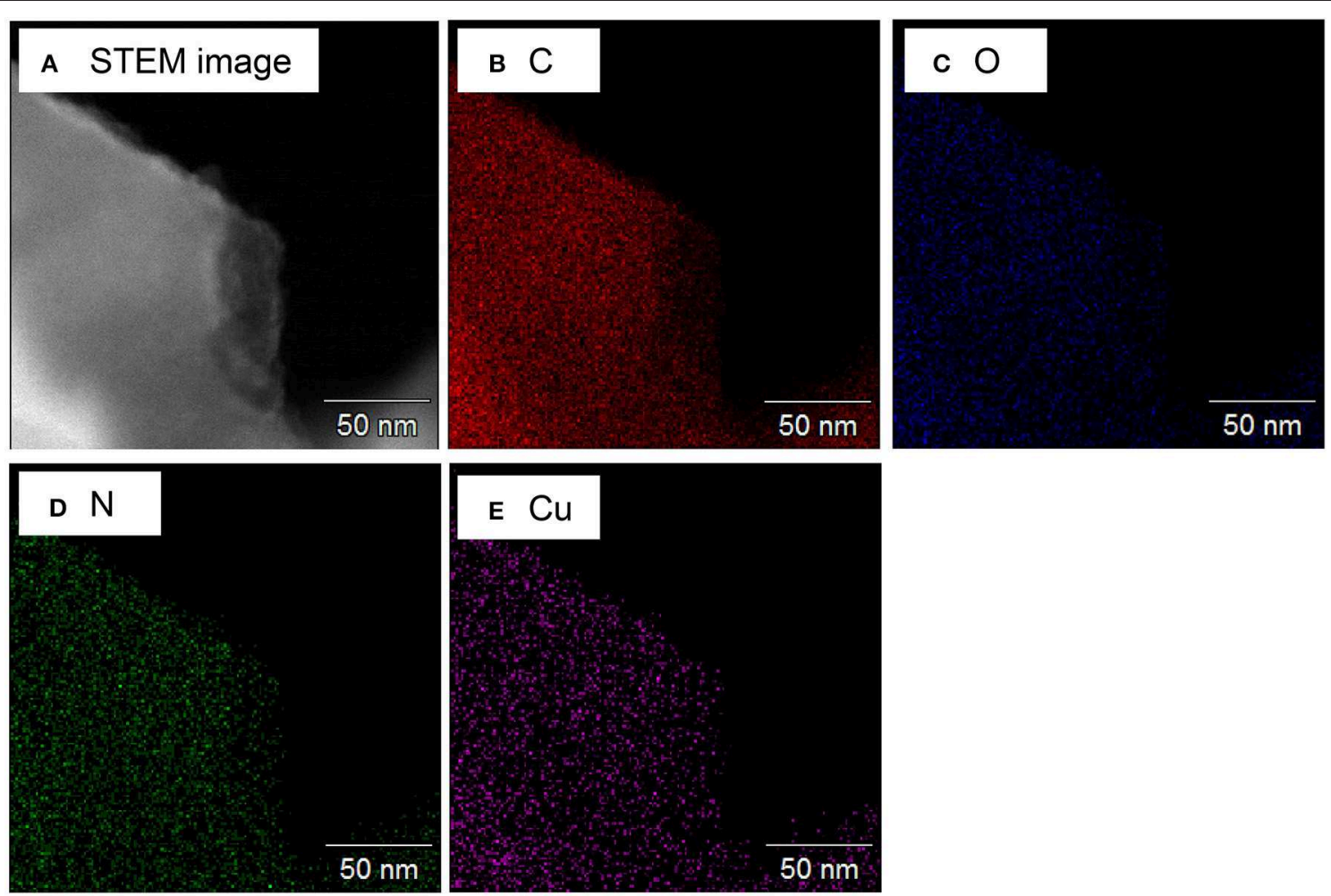

FIGURE 2 | (A) STEM-HAADF images of Cu-N-ZTC and (B-E) the elemental mapping for C, O, N, and Cu, respectively.
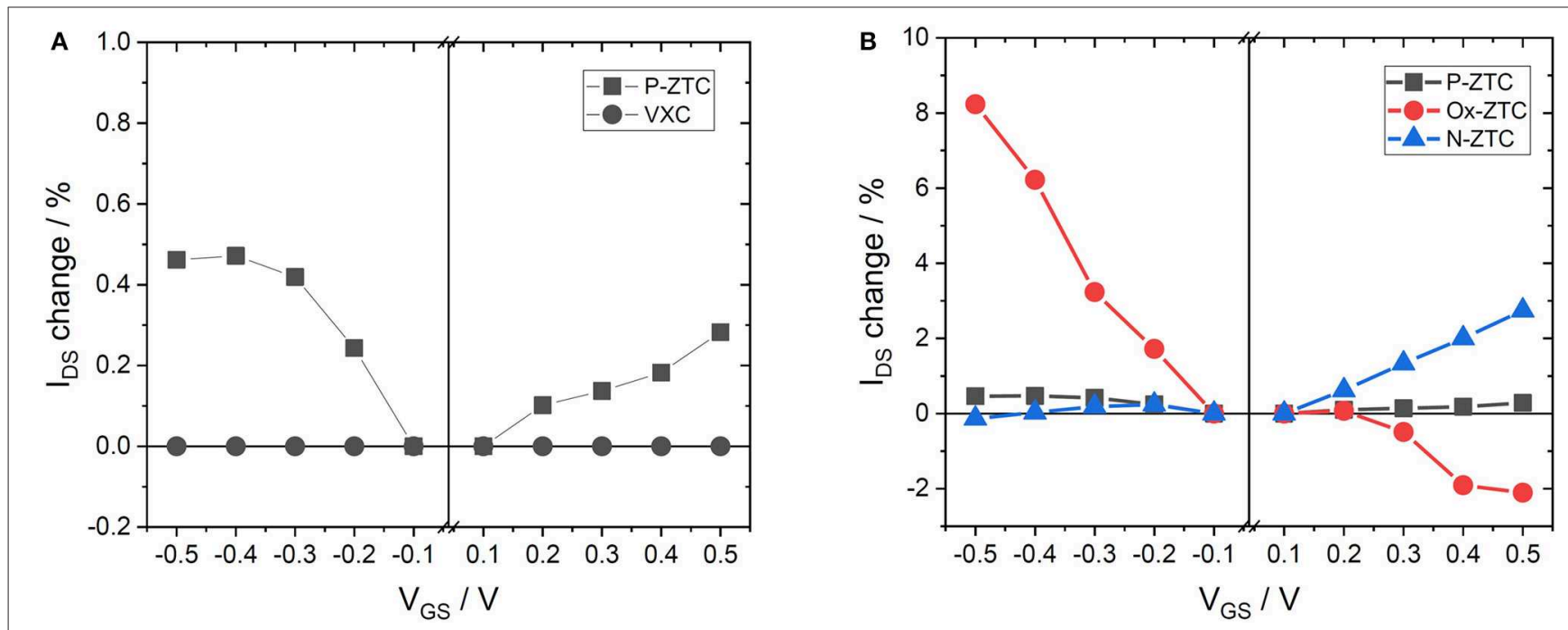

FIGURE 3 | ISFET characteristic curve of (A) P-ZTC and VXC and (B) OX-ZTC and N-ZTC.

close to the conduction band, thus the former exhibited p-type semiconductor characteristics and the latter exhibited n-type characteristics. As shown in Table 1, it has been confirmed that many oxygen-containing functional groups are formed in OxZTC. The Fermi level would be decreased because of the electron withdrawing ability of oxygen-containing functional groups. For
$\mathrm{N}$-ZTC, nitrogen species have different electronic influences on the carbon, owing to its chemical state. Therefore, the chemical state of nitrogen was analyzed from XPS measurement, and the chemical state of nitrogen was examined in more detail by performing peak deconvolution. The $\mathrm{N} 1 \mathrm{~s}$ spectrum of $\mathrm{N}$ ZTC follows the classification advocated by Raymundo-Pinero 
TABLE 3 | Work function of ZTC samples.

\begin{tabular}{lc}
\hline Sample & Work function/eV \\
\hline P-ZTC & 4.56 \\
Ox-ZTC & 4.72 \\
N-ZTC & 4.28 \\
Cu-N-ZTC & 4.69 \\
Au (as reference) & 5.10 \\
\hline
\end{tabular}

et al. (2002), i.e., pyridine (398.5 eV), pyrrol/pyridone (400.5 eV), quaternary $(401.2 \mathrm{eV})$ and oxide $(402.9 \mathrm{eV})$. Figure $\mathbf{S} 3$ shows the result of peak deconvolution of the $\mathrm{N}$ 1s spectrum of N-ZTC. These four kinds of nitrogen species are present in the same proportions in N-ZTC. According to the study by Wang et al. a doping of quaternary nitrogen promotes n-type semiconductor characteristics, and pyridine-type nitrogen generally promotes p-type characteristics (Wang et al., 2014). However, there is a report that the pyridine present in CVD graphene (Luo et al., 2011) and nanographene (Yan et al., 2007) promotes n-type semiconductor properties, and this effect is considered to be caused by hydrogenation of pyridine-type nitrogen (Schiros et al., 2012). The Fermi level of N-ZTC would be increased because of the influence of quaternary and pyridine-type nitrogen in its structure.

\section{Ion Sensing Characteristics of Cu-Doped ZTCs}

Figure 4 shows the results of the $\mathrm{V}_{\mathrm{GS}}-\mathrm{I}_{\mathrm{DS}}$ plot of the $\mathrm{Cu}$ doped ZTC samples. The ISFET characteristics of $\mathrm{Cu}$-ZTC were not significantly different from P-ZTC. In contrast, Cu-N-ZTC showed a bipolarity characteristic, and the sensitivity reached approximately $50 \%$ in $\mathrm{I}_{\mathrm{DS}}$ change $\left(\mathrm{V}_{\mathrm{GS}}=-0.5 \mathrm{~V}\right)$. This ISFET sensitivity is much higher than the reported value of GNR (Zhang and Cui, 2013). As there was a little change in sensing sensitivity with the addition of copper into ZTC, the enhancement of the sensitivity is considered to be caused by the interaction between nitrogen and copper.

In order to investigate the influence on the ZTC by the simultaneous addition of nitrogen and copper, the electronic state was considered from the work function and the chemical state of the heteroatoms in its structure. Despite the nitrogen doping of $\mathrm{Cu}-\mathrm{N}-\mathrm{ZTC}$, its work function was $4.69 \mathrm{eV}$ (as shown in Table 3), which was close to the value of Ox-ZTC. This indicates that the Fermi level was decreased by co-doping nitrogen and copper. The surface chemical states were analyzed from XPS measurement. Figure 5A shows the $\mathrm{N} 1 \mathrm{~s}$ spectra of N-ZTC and Cu-N-ZTC. Bimodal peaks were confirmed in both cases. As compared with $\mathrm{N}-\mathrm{ZTC}, \mathrm{Cu}-\mathrm{N}-\mathrm{ZTC}$ shows that the peak intensity derived from pyridine-type nitrogen on the low-energy side decreases, and the higher energy peak increases. Figure 5B shows the $\mathrm{Cu} 2 \mathrm{p}$ spectra of $\mathrm{Cu}-\mathrm{ZTC}$ and $\mathrm{Cu}-\mathrm{N}-\mathrm{ZTC}$. In $\mathrm{Cu}-\mathrm{ZTC}$, a peak attributable to $\mathrm{Cu}^{2+}(\mathrm{CuO})$ near $934.5 \mathrm{eV}$ was confirmed. In contrast, in $\mathrm{Cu}-$ $\mathrm{N}$-ZTC, the $\mathrm{Cu} 2 \mathrm{p}$ peak position shifted to a lower-energy side than that in $\mathrm{Cu}-\mathrm{ZTC}$, which was attributed to $\mathrm{Cu}^{+}(932.4 \mathrm{eV})$.

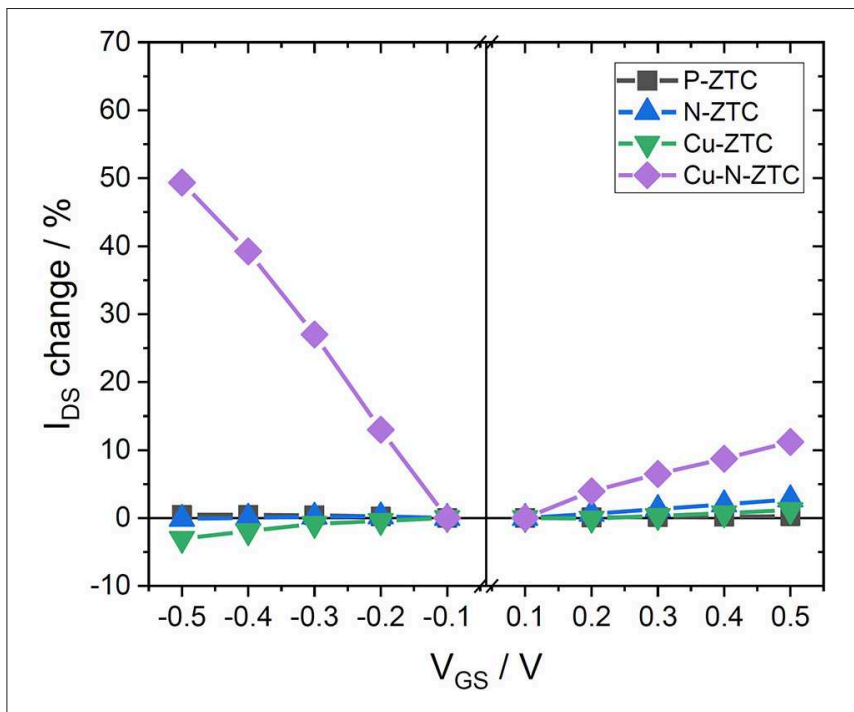

FIGURE 4 | ISFET characteristic curve of Cu doped ZTCs.

Shaozheng and colleagues have reported that the presence of $\mathrm{Cu}-\mathrm{N}$ bond results in a down shift of the $\mathrm{Cu} 2 \mathrm{p}$ peak from $\mathrm{Cu}^{2+}$ to $\mathrm{Cu}^{+}$simultaneously with the upshift of the energy of $\mathrm{N}$ 1s peak (Hu et al., 2017). This is consistent with the change in $\mathrm{N} 1 \mathrm{~s}$ and the $\mathrm{Cu} 2 \mathrm{p}$ peak energy shift seen in Figure 5, suggesting that $\mathrm{Cu}-\mathrm{N}$ bond present in the structure, and that nitrogen species coordinated with copper ion certainly exist. The peak appeared at $400.5 \mathrm{eV}$ in the $\mathrm{N} 1$ s difference spectrum (shown in Figure 5A) with attributes of the $\mathrm{N}$ species bonding with $\mathrm{Cu}^{+}$. From this complex formation of $\mathrm{Cu}^{+}$and nitrogen, we can explain the decrease of the Fermi level of Cu-N-ZTC. When nitrogen species are coordinated by $\mathrm{Cu}^{+}$, electrons of the ligand nitrogen are attracted to the $\mathrm{Cu}^{+}$. Concomitantly, nitrogen acquires an electron withdrawing ability, and attracts $\pi$ electrons on the graphenic structure. From the above discussion, the formation of the complex of nitrogen and $\mathrm{Cu}^{+}$is considered to shift the Fermi level to the lower-energy side.

In the previous section, the formation of coordination of nitrogen and copper showed that the Fermi level moved to the low-energy side. However, considering only the movement of the Fermi level, $\mathrm{Cu}-\mathrm{N}$-ZTC should show a p-type semiconductor characteristic that is the same as that in the case of Ox-ZTC. In order to investigate the cause of the exhibited bipolarity shown in $\mathrm{Cu}-\mathrm{N}-\mathrm{ZTC}$, the effect of electrochemical potential on the state of $\mathrm{Cu}$ was evaluated. The electrochemical potential of $\mathrm{Cu}-\mathrm{N}-\mathrm{ZTC}$ was set at a predetermined potential, and the XPS measurement of the sample was immediately started. As the open circuit potential (OCP) of the $\mathrm{Cu}-\mathrm{N}-\mathrm{ZTC}$ is approximately $0.65 \mathrm{~V}$ vs. $\mathrm{RHE}$ in $1 \mathrm{~mol} \mathrm{~L}^{-1} \mathrm{HCl}$ aqueous solution, the potential sweep range was set to 0.4 to $0.9 \mathrm{~V}$ vs. RHE. Figure 6 shows the $\mathrm{Cu} 2 \mathrm{p}$ spectrum of $\mathrm{Cu}-\mathrm{N}-\mathrm{ZTC}$ at OCP, reduction potential $(0.4 \mathrm{~V}$ vs. RHE), and oxidation potential $(0.9 \mathrm{~V}$ vs. $\mathrm{RHE})$. The $\mathrm{Cu} 2 \mathrm{p}$ peak shifted by approximately $1 \mathrm{eV}$ from the oxidation state to reduction state, indicating that the chemical state of copper in $\mathrm{Cu}-\mathrm{N}-\mathrm{ZTC}$ varies with the 

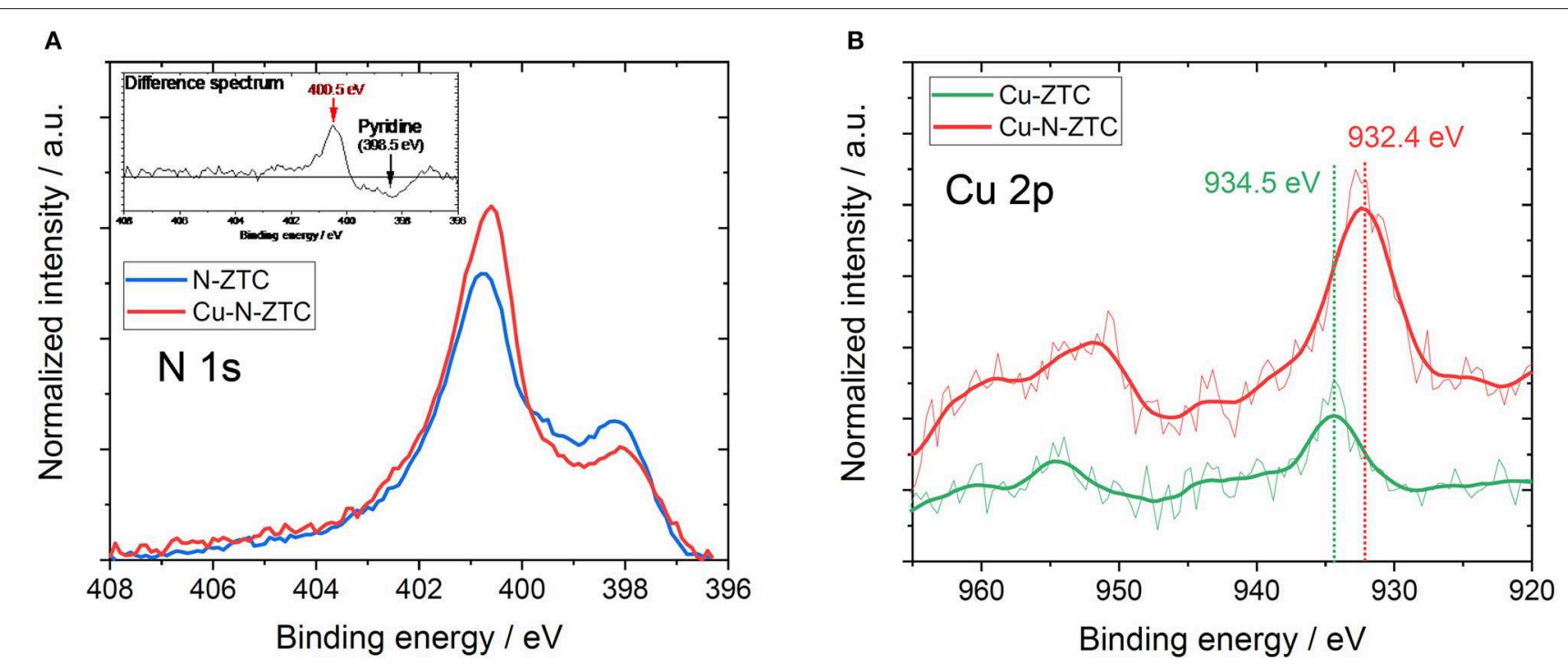

FIGURE 5 | XPS spectra of (A) N 1s region and (B) Cu 2p region of ZTC samples.

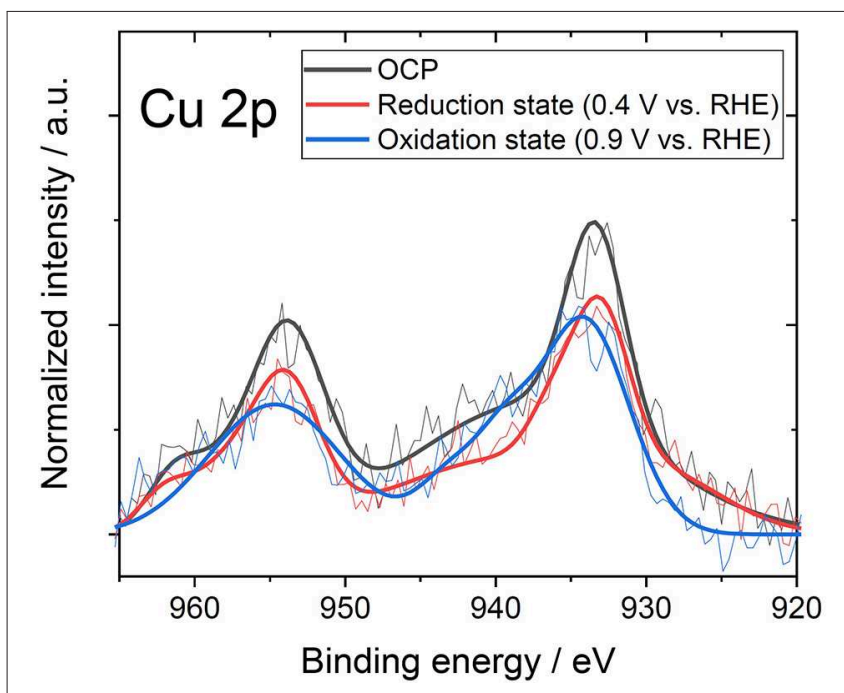

FIGURE 6 | XPS spectra of Cu 2p region for Cu-N-ZTCs being in different redox states.

electrochemical potential, and exists in a redoxable state. The bipolarity characteristics $\mathrm{Cu}-\mathrm{N}-\mathrm{ZTC}$ shown can be explained based on the above electrochemical evaluation. Owing to the changing chemical state of copper along with the $\mathrm{V}_{\mathrm{GS}}$, the degree of the electronic distribution between the nitrogen and copper would be changed. This polarization change affects the electron withdrawing ability of the coordination of nitrogen and copper toward $\pi$-electrons of carbon, and as a result, induces bipolarity in its ISFET characteristic.

It is conceivable that the sensitivity of $\mathrm{Cu}-\mathrm{N}-\mathrm{ZTC}$ in ISFET is enhanced by physical and chemical effects. In the physical adsorption of ions that is the common case in ISFET, the electron carrier is supplemented with electrostatic force. In contrast, the change of electronic polarization between nitrogen and copper is supposed to chemically supplement the carrier of the $\pi$-electron system. The former is a physical field effect, and the latter can be regarded as a chemical change of a functional group. It is considered that $\mathrm{Cu}-\mathrm{N}-\mathrm{ZTC}$ showed high sensing sensitivity owing to the effects of both. From the above considerations, the change in the electronic polarization between nitrogen and copper by the applied $\mathrm{V}_{\mathrm{GS}}$ causes the bipolarity of the ISFET characteristic and the enhancement of the sensitivity.

\section{CONCLUSION}

In this study, we focused on ZTC composed of a threedimensional network of GNR as an alternative to GNR and evaluated its ISFET characteristics. Various heteroatoms such as oxygen, nitrogen, and copper were doped in the structure of ZTC, and the carbon structure of ZTC was maintained even when doping them. The evaluation of ISFET characteristics of various ZTC samples showed that the un-doped ZTC exhibited bipolar characteristics, whereas the oxygen- and nitrogendoped samples showed p-type and n-type semiconductor characteristics, respectively. The co-doped sample of $\mathrm{Cu}$ and $\mathrm{N}$ (Cu-N-ZTC) showed bipolarity as an ISFET characteristic, and exhibited the highest sensitivity. The detailed investigation confirmed that the coordination of nitrogen and copper exists in the structure of $\mathrm{Cu}-\mathrm{N}-\mathrm{ZTC}$, and the chemical state of copper can be changed by an electrochemical potential. The specific ISFET characteristic of $\mathrm{Cu}-\mathrm{N}-\mathrm{ZTC}$ was explained by the existence of the coordination. Regarding the interaction between carbon and heteroatoms, its essence is quite deep, 
and still insufficiently understood. This study recognizes the influence of heteroatoms, and particularly the coordination of nitrogen and copper, on the electronic state of carbon and ISFET characteristics. Furthermore, the manner of interaction between carbon and heteroatoms could be developed based on the evaluation of ISFET characteristics.

\section{DATA AVAILABILITY}

All datasets generated for this study are included in the manuscript and/or the Supplementary Files.

\section{AUTHOR CONTRIBUTIONS}

All authors listed have made a substantial, direct and intellectual contribution to the work, and approved it for publication.

\section{REFERENCES}

Ahmadi, M. T., Ismail, R., and Anwar, S. (2016). Handbook of Research on Nanoelectronic Sensor Modeling and Applications. Hershey, PA: IGI Global.

Bai, J. W., and Huang, Y. (2010). Fabrication and electrical properties of graphene nanoribbons. Mater. Sci. Eng. $R$ Rep. 70, 341-353. doi: 10.1016/j.mser.2010.06.019

Berenguer-Murcia, Á., Ruiz-Rosas, R. R., García-Aguilar, J., Nueangnoraj, K., Nishihara, H., Morallón, E., et al. (2013). Binderless thin films of zeolitetemplated carbon electrodes useful for electrochemical microcapacitors with ultrahigh rate performance. Phys. Chem. Chem. Phys. 15, 10331-10334. doi: $10.1039 / \mathrm{c} 3 \mathrm{cp} 51945 \mathrm{~g}$

Bronner, C., Leyssner, F., Stremlau, S., Utecht, M., Saalfrank, P., Klamroth, T., et al. (2012). Electronic structure of a subnanometer wide bottom-up fabricated graphene nanoribbon: End states, band gap, and dispersion. Phys. Rev. B 86:085444. doi: 10.1103/PhysRevB.86.085444

Chen, X., Liu, Y., Fang, X., Li, Z., Pu, H., Chang, J., et al. (2019). Ultratrace antibiotic sensing using aptamer/graphene-based field-effect transistors. Biosens. Bioelectron. 126, 664-671. doi: 10.1016/j.bios.2018.11.034

Chen, X., Pu, H., Fu, Z., Sui, X., Chang, J., Chen, J., et al. (2018). Real-time and selective detection of nitrates in water using graphene-based field-effect transistor sensors. Environ. Sci. Nano 5, 1990-1999. doi: 10.1039/C8EN00588E

Deng, Y. F., Xie, Y., Zou, K. X., and Ji, X. L. (2016). Review on recent advances in nitrogen-doped carbons: preparations and applications in supercapacitors. $J$. Mater. Chem. A 4, 1144-1173. doi: 10.1039/C5TA08620E

Duroux, P., Emde, C., Bauerfeind, P., Francis, C., Grisel, A., Thybaud, L., et al. (1991). The ion sensitive field-effect transistor (Isfet) Ph Electrode a new sensor for long-term ambulatory Ph monitoring. Gut 32, 240-245. doi: 10.1136/gut.32.3.240

Hoshikawa, Y., An, B. G., Kashihara, S., Ishii, T., Ando, M., Fujisawa, S., et al. (2016). Analysis of the interaction between rubber polymer and carbon black surfaces by efficient removal of physisorbed polymer from carbon-rubber composites. Carbon 99, 148-156. doi: 10.1016/j.carbon.2015.12.003

Hu, S. Z., Qu, X. Y., Bai, J., Li, P., Li, Q., Wang, F., et al. (2017). Effect of $\mathrm{Cu}(\mathrm{I})-\mathrm{N}$ active sites on the $\mathrm{N}-2$ photofixation ability over flowerlike copperdoped g-C3N4 prepared via a novel molten salt-assisted microwave process: the experimental and density functional theory simulation analysis. Acs Sustain. Chem. Eng. 5, 6863-6872. doi: 10.1021/acssuschemeng.7b01089

Ishii, T., Kaburagi, Y., Yoshida, A., Hishiyama, Y., Oka, H., Setoyama, N., et al. (2017a). Analyses of trace amounts of edge sites in natural graphite, synthetic graphite and high-temperature treated coke for the understanding of their carbon molecular structures. Carbon 125, 146-155. doi: 10.1016/j.carbon.2017.09.049

Ishii, T., Kashihara, S., Hoshikawa, Y., Ozaki, J., Kannari, N., Takai, K., et al. (2014). A quantitative analysis of carbon edge sites and an estimation of graphene sheet

\section{ACKNOWLEDGMENTS}

We acknowledge the financial support by Advanced Low Carbon Technology Research and Development Program of the Japan Science and Technology Agency and Ministry of Education, Science, Sports and Culture, Grant-in-Aid for Scientific Research, 18K14312. This work has been also supported by Izumi Science and Technology Foundation. STEM-HAADF measurement was carried out at National Institute for Materials Science (NIMS) Battery Research Platform.

\section{SUPPLEMENTARY MATERIAL}

The Supplementary Material for this article can be found online at: https://www.frontiersin.org/articles/10.3389/fmats. 2019.00129/full\#supplementary-material

size in high-temperature treated, non-porous carbons. Carbon 80, 135-145. doi: 10.1016/j.carbon.2014.08.048

Ishii, T., Maie, T., Kimura, N., Kobori, Y., Imashiro, Y., and Ozaki, J. (2017b). Enhanced catalytic activity of nanoshell carbon co-doped with boron and nitrogen in the oxygen reduction reaction. Int. J. Hydrogen Energy 42, 15489-15496. doi: 10.1016/j.ijhydene.2017.05.003

Itoi, H., Nishihara, H., Ishii, T., Nueangnoraj, K., Berenguer-Betrian, R., and Kyotani, T. (2014). Large pseudocapacitance in quinonefunctionalized zeolite-templated carbon. Bull. Chem. Soc. Japan 87, 250-257. doi: 10.1246/bcsj.20130292

Itoi, H., Nishihara, H., Kobayashi, S., Ittisanronnachai, S., Ishii, T., Berenguer, R., et al. (2017). Fine dispersion of Pt4-5 subnanoclusters and Pt single atoms over porous carbon supports and their structural analyses with X-ray absorption spectroscopy. J. Phys. Chem. C 121, 7892-7902. doi: 10.1021/acs. jpcc.7b00422

Itoi, H., Nishihara, H., Kogure, T., and Kyotani, T. (2011). Three-dimensionally arrayed and mutually connected 1.2-nm nanopores for high-performance electric double layer capacitor. J. Am. Chem. Soc. 133, 1165-1167. doi: $10.1021 / \mathrm{ja} 108315 \mathrm{p}$

Itoi, H., Nishihara, H., and Kyotani, T. (2016). Effect of heteroatoms in ordered microporous carbons on their electrochemical capacitance. Langmuir 32, 11997-12004. doi: 10.1021/acs.langmuir.6b02667

Kahn, A. (2016). Fermi level, work function and vacuum level. Mater. Horizons 3, 7-10. doi: 10.1039/C5MH00160A

Kan-Nari, N., Okamura, S., Fujita, S., Ozaki, J., and Araib, M. (2010). Nitrogendoped carbon materials prepared by ammoxidation as solid base catalysts for knoevenagel condensation and transesterification reactions. Adv. Synth. Catal. 352, 1476-84. doi: 10.1002/adsc.201000029

Kashihara, S., Otani, S., Orikasa, H., Hoshikawa, Y., Ozaki, J., and Kyotani, T. (2012). A quantitative analysis of a trace amount of hydrogen in high temperature heat-treated carbons. Carbon 50, 3310-3314. doi: 10.1016/j.carbon.2011.12.029

Kosynkin, D. V., Higginbotham, A. L., Sinitskii, A., Lomeda, J. R., Dimiev, A., Price, B. K., et al. (2009). Longitudinal unzipping of carbon nanotubes to form graphene nanoribbons. Nature 458, 872-U875. doi: 10.1038/nature07872

Luo, Z. Q., Lim, S. H., Tian, Z. Q., Shang, J. Z., Lai, L. F., Macdonald, B., et al. (2011). Pyridinic N doped graphene: synthesis, electronic structure, and electrocatalytic property. J. Mater. Chem. 21, 8038-8044. doi: $10.1039 / \mathrm{cljm} 10845 \mathrm{j}$

Mao, S., Chang, J., Pu, H., Lu, G., He, Q., Zhang, H., et al. (2017). Two-dimensional nanomaterial-based field-effect transistors for chemical and biological sensing. Chem. Soc. Rev. 46, 6872-6904. doi: 10.1039/C6CS00827E

Martinoia, S., Massobrio, G., and Lorenzellib, L. (2005). Modeling ISFET microsensor and ISFET-based microsystems: a review. Sens. Actuat. B Chem. 105, 14-27. doi: 10.1016/S0925-4005(04)00107-8 
Nishihara, H., Ittisanronnachai, S., Itoi, H., Li, L. X., Suzuki, K., Nagashima, U., et al. (2014). Experimental and theoretical studies of hydrogen/deuterium spillover on Pt-loaded zeolite-templated carbon. J. Phys. Chem. C 118, 9551-9559. doi: 10.1021/jp5016802

Nishihara, H., and Kyotani, T. (2018). Zeolite-templated carbons - threedimensional microporous graphene frameworks. Chem. Commun. 54, 5648-5673. doi: 10.1039/C8CC01932K

Nueangnoraj, K., Nishihara, H., Ishii, T., Yamamoto, N., Itoi, H., Berenguer, R., et al. (2015). Pseudocapacitance of zeolite-templated carbon in organic electrolytes. Energy Storage Mater. 1, 35-41. doi: 10.1016/j.ensm.2015.08.003

Ozaki, J., Anahara, T., Kimura, N., and Oya, A. (2006). Simultaneous doping of boron and nitrogen into a carbon to enhance its oxygen reduction activity in proton exchange membrane fuel cells. Carbon 44, 3358-3361. doi: 10.1016/j.carbon.2006.08.022

Raymundo-Pinero, E., Cazorla-Amoros, D., Linares-Solano, A., Find, J., Wild, U., and Schlogl, R. (2002). Structural characterization of N-containing activated carbon fibers prepared from a low softening point petroleum pitch and a melamine resin. Carbon 40, 597-608. doi: 10.1016/S0008-6223(01)00155-5

Schiros, T., Nordlund, D., Pálová, L., Prezzi, D., Zhao, L., Kim, K. S., et al. (2012). Connecting dopant bond type with electronic structure in $\mathrm{N}$-doped graphene. Nano Lett. 12, 4025-4031. doi: 10.1021/nl301409h

Syu, Y. C., Hsu, W. E., and Lin, C. T. (2018). Review-field-effect transistor biosensing: devices and clinical applications. Ecs J. Solid State Sci. Technol. 7, Q3196-Q3207. doi: 10.1149/2.0291807jss
Wang, X., Sun, G., Routh, P., Kim, D. H., Huang, W., and Chen, P. (2014). Heteroatom-doped graphene materials: syntheses, properties and applications. Chem. Soc. Rev. 43, 7067-7098. doi: 10.1039/C4CS00141A

Yan, Q., Huang, B., Yu, J., Zheng, F., Zang, J., Wu, J., et al. (2007). Intrinsic currentvoltage characteristics of graphene nanoribbon transistors and effect of edge doping. Nano Lett. 7, 1469-1473. doi: 10.1021/nl070133j

Yu, S. S., Zheng, W. T., and Jiang, Q. (2010). Electronic properties of nitrogen/boron-doped graphene nanoribbons with armchair edges. IEEE Trans. Nanotechnol. 9, 78-81. doi: 10.1109/TNANO.2009.2020797

Zhang, B., and Cui, T. H. (2013). Suspended graphene nanoribbon ionsensitive field-effect transistors formed by shrink lithography for $\mathrm{pH} /$ cancer biomarker sensing. J. Microelectromech. Syst. 22, 1140-1146. doi: 10.1109/JMEMS.2013.2254701

Conflict of Interest Statement: The authors declare that the research was conducted in the absence of any commercial or financial relationships that could be construed as a potential conflict of interest.

Copyright (c) 2019 Ishii, Horiuchi and Ozaki. This is an open-access article distributed under the terms of the Creative Commons Attribution License (CC BY). The use, distribution or reproduction in other forums is permitted, provided the original author(s) and the copyright owner(s) are credited and that the original publication in this journal is cited, in accordance with accepted academic practice. No use, distribution or reproduction is permitted which does not comply with these terms. 